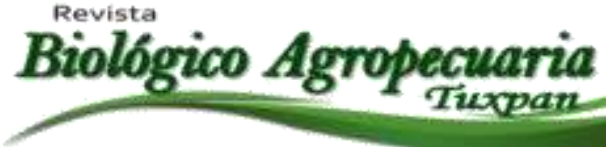

\title{
Variación de la precipitación y temperatura en el municipio de Teziutlán, Puebla
}

Variation of precipitation and temperature in the municipality of Teziutlán, Puebla

${ }^{\square}$ Villegas Rodríguez Isaac ${ }^{1}$, José Méndez Gómez ${ }^{1}$, Delfino Reyes López ${ }^{1}$, Benjamín Barrios Díaz ${ }^{1}$ y Cesar David Torres Fernandez ${ }^{1}$

${ }^{1}$ Profesor-investigador. ${ }^{2}$ Alumno. Benemérita Universidad Autónoma de Puebla. Facultad de Ingeniería Agrohidráulica. San Juan Acateno, Teziutlán, Puebla, México. Tel. 2313122933.

${ }^{\square}$ Autor para correspondencia: isaacvillegasr@hotmail.com.mx

Recibido: 06/01/2014

Aceptado: 12/07/2014

\section{RESUMEN}

El municipio de Teziutlán se ubica en la zona nororiente del estado de Puebla, se caracteriza por tener un clima templado húmedo con lluvias distribuidas generalmente todo el año, sin embargo en los últimos años se ha observado que hay un cambio en cuánto a la precipitación y temperatura, teniendo como referencia las inundaciones y deslaves en el municipio de Teziutlán, causando pérdidas económicas en el sector agropecuario e incluso pérdidas humanas. En este trabajo se analizó los datos estadísticos en bloques de 10 años para observar la variación de la precipitación y temperatura. Los resultado indican que el periodo de lluvia se ha desfasado un mes y la temperatura se ha incrementado entre 0.5 a $1.5^{\circ} \mathrm{C}$., tomando en cuenta las estadísticas marcan que entre 9 y 11 años hay un periodo de retorno de precipitación y esquiaje donde la precipitación anual varía de1500mm a 3600 mm.

Palabras clave: Clima, Precipitación, Temperaturas, Estación Meteorológica y Estaciones del año.

\begin{abstract}
Teziutlán Township is located in the northeast of the state of Puebla, is characterized by a humid temperate climate with rain generally spread throughout the year, however in recent years have shown that there is a change in how precipitation and temperature, referencing the floods and landslides in the
\end{abstract}


town of Teziutlán, causing economic losses in agriculture and even human losses. In this paper we analyzed statistical data in blocks of 10 years to observe the variation in precipitation and temperature. The results indicate that the period of rain has lagged one month and the temperature has increased by 0.5 to $1.5^{\circ} \mathrm{C}$. Taking into account the statistics make that between 9 and 11 years there is a return period of precipitation and skiing where annual rainfall averages $3600 \mathrm{~mm}$ of $1500 \mathrm{~mm}$.

Keywords: Climate, Precipitation, Temperature, Weather Station and Seasons.

\section{INTRODUCCIÓN}

En los municipios del estado de Puebla, se tiene información de las dependencias gubernamentales: Comisiona Nacional del Agua (CNA) y Comisión Federal de

Electricidad (CFE) tienen estaciones meteorologías a su cargo para registrar todos los días a las 8:0 a.m. los datos de precipitación, evaporación, temperaturas máximas y mínimas, dirección y velocidad del viento. El clima se puede definir como el conjunto de factores atmosféricos que permiten que las diversas regiones de nuestro globo sean adecuadas para mantener la vida humana, animal y vegetal. (Ayllón, 1996). El municipio se localiza en la transición de los climas templados de la Sierra Norte, a los cálidos del declive del golfo; se identifican tres climas: C (m). Clima templado húmedo con abundantes lluvias en verano. Se presenta en una pequeña área del extremo sur del municipio, $14.15 \%$ de la superficie municipal; C (fm). Clima templado húmedo con lluvias todo el año. Se intensifica en una franja latitudinal al centro y sur del Municipio que representa el $60.82 \%$ del territorio; (A) C

(fm). Clima semicalido-húmedo, con abundantes lluvias todo el año. Se presenta en el Norte del Municipio en el $25.03 \%$ de su superficie (INEGI, 2000). Determinar el comportamiento histórico de la precipitación y la temperatura promedio mensual en un periodo de treinta años mediante, el análisis estadístico de las variables meteorológicas en bloque de años, permitirá conocer si hay cambios, y de que magnitud modificando.

\section{MATERIALES Y MÉTODOS}

En el municipio de la estación meteorológica que se encuentra ubicada en escuela de Ingeniería Agrohidráulica, de la Benemérita Universidad Autónoma de Puebla en la Junta Auxiliar de San Juan Acateno, es la única de las estaciones que se encuentra en funcionamiento (Fig. 1).

Para realizar este trabajo se tomó en cuenta 30 años de registro de una sola estación para minimizar el error en la base de datos, los datos se dividió en tres bloques de 10 años cada uno se determinará el promedio de la precipitación, temperatura máxima y mínima en los tres bloques y se compara con la media general de los 30 años y saber su comportamiento de cada bloque. La ubicación de una estación es de gran importancia que esta cuente con una superficie despejada que por lo menos los árboles este retirado de $25 \mathrm{o} 30 \mathrm{~m}$, que cuente con una malla de protección perimetral.

\section{Revista Científica Biológico Agropecuaria Tuxpan 2 (1) ISSN: 2007-6940}




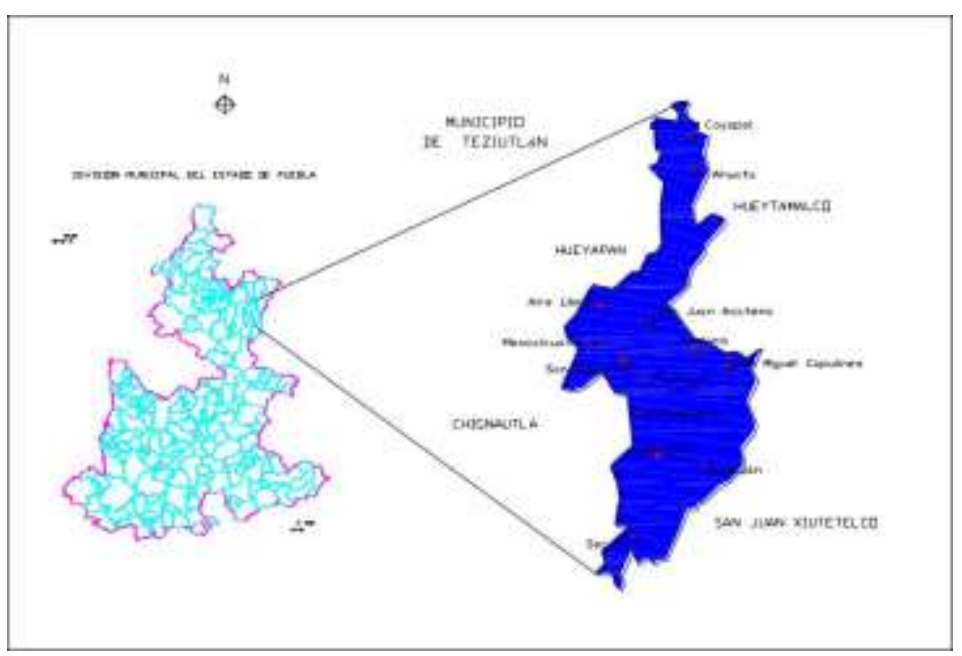

Figura 1. Localización geográfica de área d estudio.

\section{RESULTADOS Y DISCUSIÓN}

El bloque de estudio 1979 al 2008 la temperatura promedio máxima oscila entre $24^{\circ} \mathrm{C}$ y $30^{\circ} \mathrm{C}$ donde el periodo de primavera la temperatura oscila entre $\operatorname{los} 28^{\circ} \mathrm{C}$ y $29.6^{\circ} \mathrm{C}$, posteriormente en las estaciones restantes la temperatura varia de $24^{\circ} \mathrm{C}$ a $25^{\circ} \mathrm{C}$. En el primer bloque 1979-1988 la temperatura en el periodo primavera oscila entre los $29.6^{\circ} \mathrm{C}$ y $30.3^{\circ} \mathrm{C}$ y el resto del año la temperatura fue de $24^{\circ} \mathrm{C}$ y $25^{\circ} \mathrm{C}$. Es muy semejante a la temperatura promedio de todos los años en estudio. De acuerdo al segundo bloque 1989-1998 durante el periodo de primavera la temperatura máxima muestran un decremento de $1^{\circ} \mathrm{C}$ con respecto al promedio total. Con lo que respecta al tercer bloque 1999-2008 la temperatura máxima empiezan a marcarse a partir del mes de febrero al mes de mayo, donde los meses restantes registran una temperatura máxima promedio entre $25.1^{\circ} \mathrm{C}$ a $25.8^{\circ} \mathrm{C}$. Con respecto al promedio total nos muestra un incremento de $1^{\circ} \mathrm{C}$ (Fig. 2). 


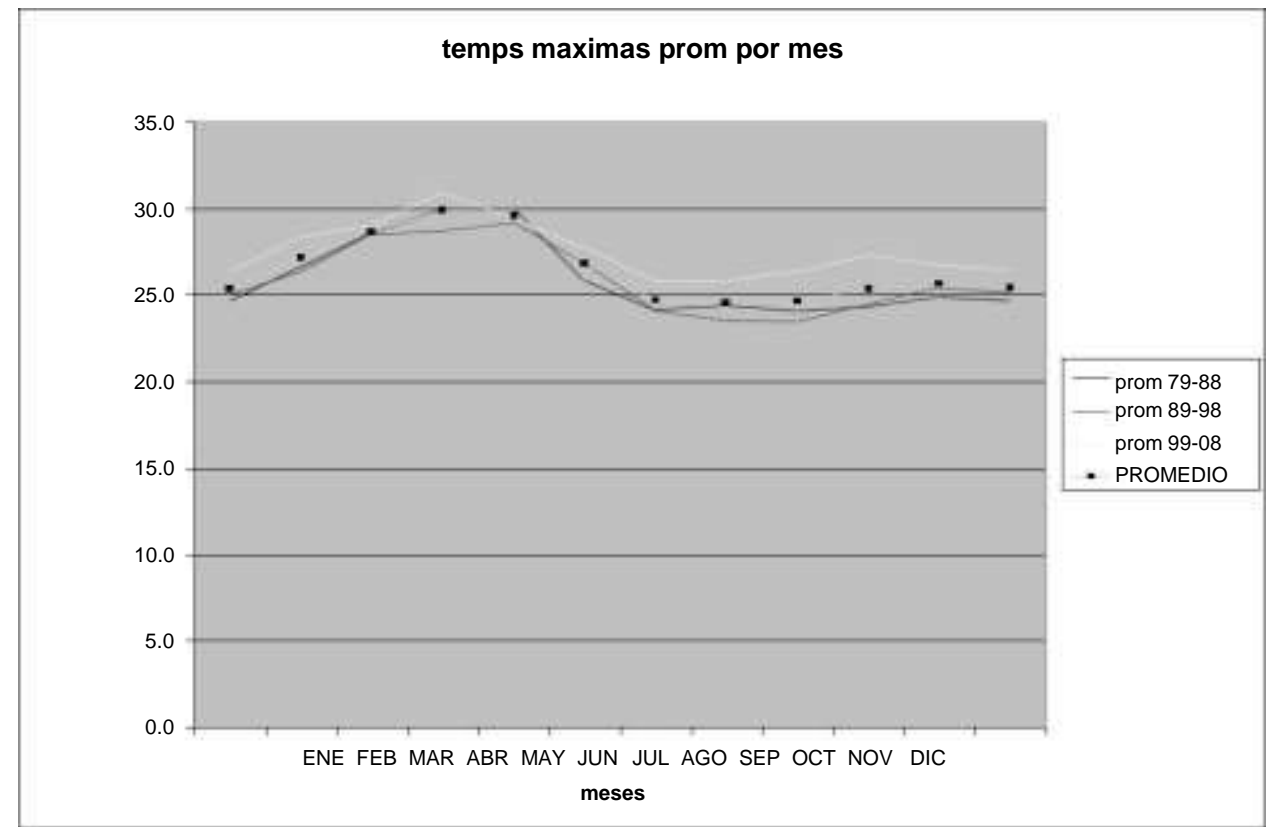

Figura 2. Temperatura Máxima Promedio por Bloques de 1979 - 2008 Años. La precipitación acumulada por año desde 1979- 2008 (Fig. 3). 


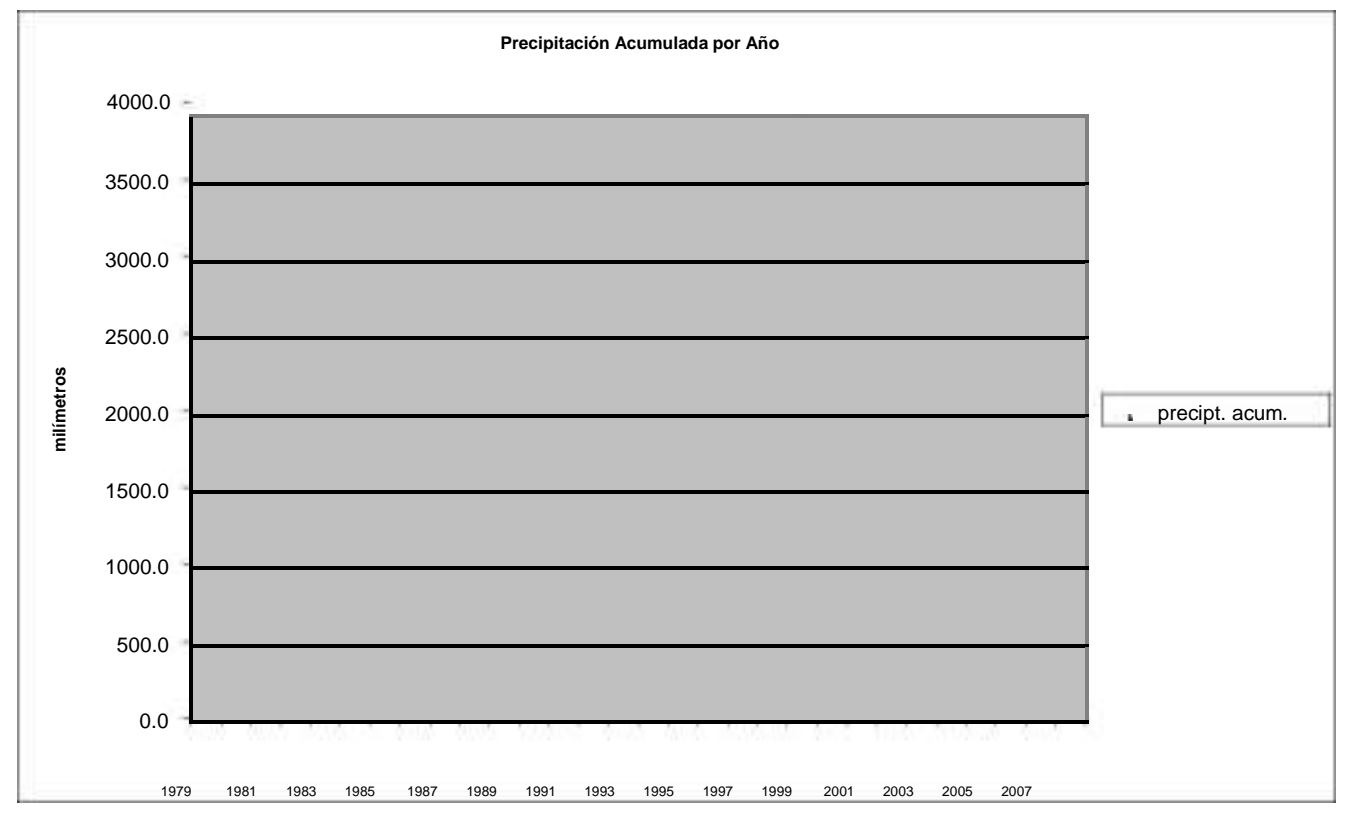

Figura 3. Precipitación por bloque de diez años.

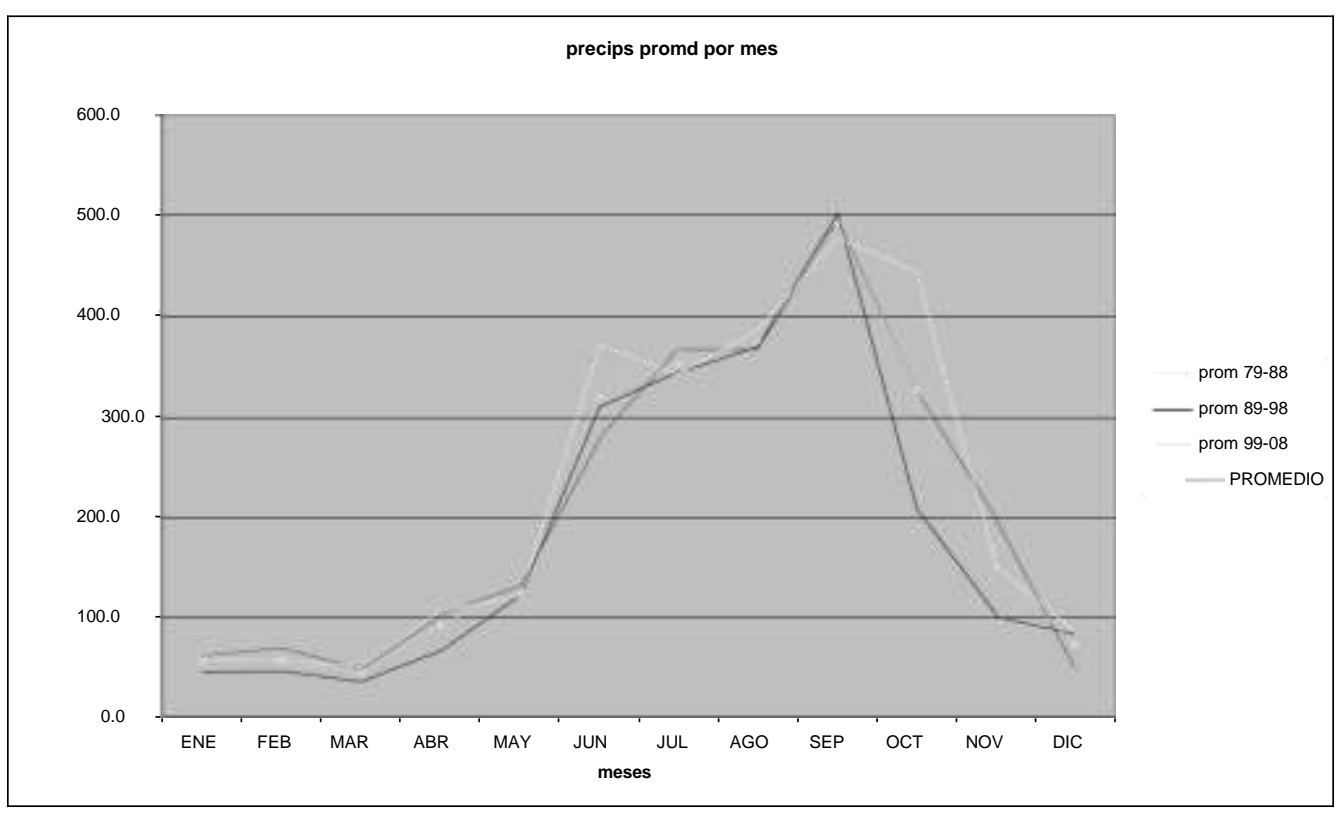

Figura 4. La temperatura media anual en municipio de Teziutlán. 
La temperatura promedio en la región varía de $14^{\circ}$ a $20^{\circ}$ grados, actualmente la temperatura mínima promedio es de $7^{\circ} \mathrm{y}$ temperatura máxima promedio es de $30^{\circ}$ (Fig. $5)$.

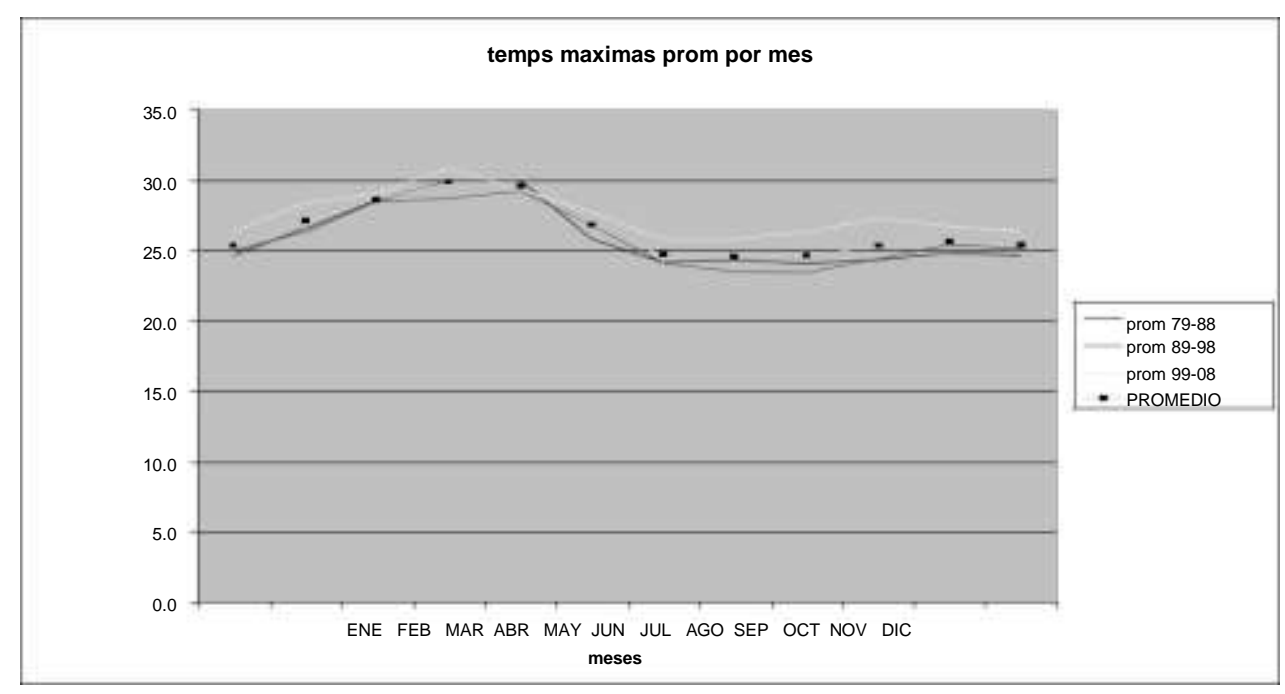

Figura 5. Temperatura Máxima Promedio por Bloques de 1979 - 2008 años.

En esta grafica se observa que el bloque de estudio 1979 al 2008 la temperatura promedio máxima oscila entre $24^{\circ} \mathrm{C}$ y $30^{\circ} \mathrm{C}$ donde el periodo de primavera la temperatura oscila entre $\operatorname{los} 28^{\circ} \mathrm{C}$ y $29.6^{\circ} \mathrm{C}$, posteriormente en las estaciones restantes la temperatura varia de $24^{\circ} \mathrm{C}$ a $25^{\circ} \mathrm{C}$. En el primer bloque 1979 1988 la temperatura en el periodo primavera oscila entre los $29.6^{\circ} \mathrm{C}$ y $30.3^{\circ} \mathrm{C}$ y el resto del año la temperatura fue de $24^{\circ} \mathrm{C}$ y $25^{\circ} \mathrm{C}$. Es muy semejante a la temperatura promedio de todos los años en estudio. De acuerdo al segundo bloque 1989-1998 durante el periodo de primavera la temperatura máxima muestran un decremento de $1{ }^{\circ} \mathrm{C}$ con respecto al promedio total. Con lo que respecta al tercer bloque 19992008 la temperatura máxima empiezan a marcarse a partir del mes de febrero al mes de mayo, donde los meses restantes registran una temperatura máxima promedio entre $25.1^{\circ} \mathrm{C}$ a $25.8^{\circ} \mathrm{C}$. Con respecto al promedio total nos muestra un incremento de $1^{\circ} \mathrm{C}$ (Fig. 6). 


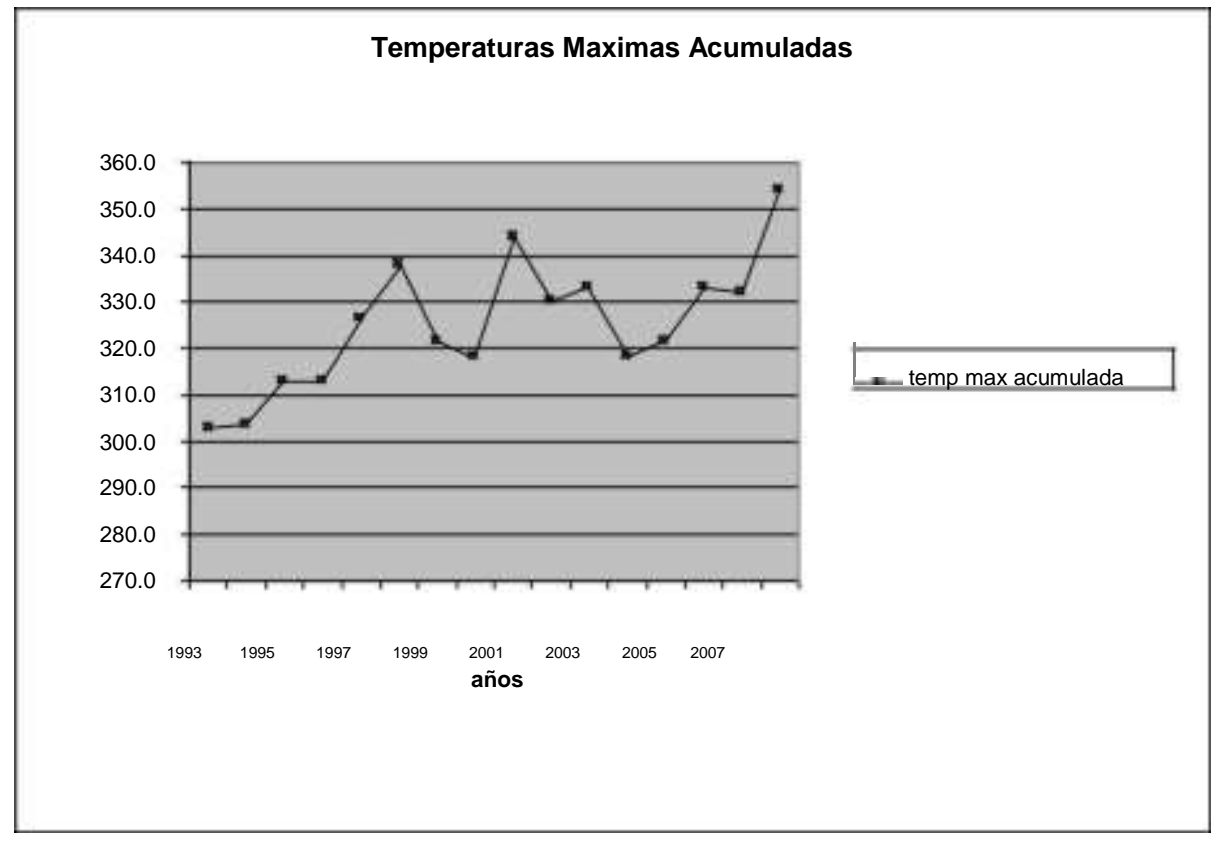

Figura 6. Temperaturas Máximas Acumuladas en los años 1993 - 2008.

La gráfica muestra que a partir del año 1992 a 2008 si la hubo. El rango en la temperatura es 320 a 340 En lo concerniente a los años de 1999 al 2008, la temperatura acumulada tiene incrementos por año de $4^{\circ} \mathrm{C}$ en promedio (Fig. 7). 


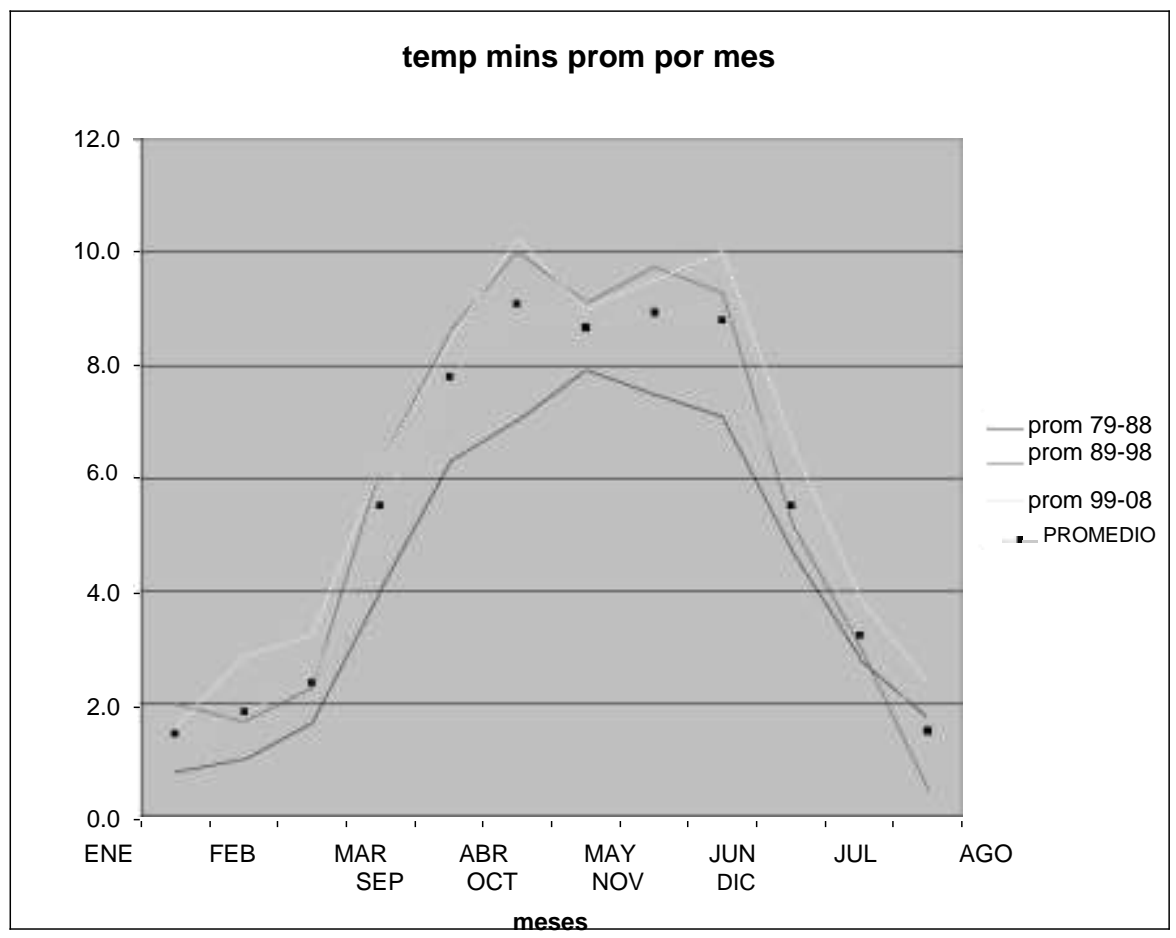

Figura 7. Temperatura Mínima Promedio en años en bloque de 1979 -2008.

La temperatura mínima por mes oscila de $1^{\circ} \mathrm{C}$ a $10^{\circ} \mathrm{C}$ observando que la temperatura mínima nos marca los cambios que se muestran en cada estación. La temperatura mínima para el periodo de primavera comienza en $2^{\circ} \mathrm{C}$ y se va incrementando por mes $2^{\circ} \mathrm{C}$, manteniéndose en el verano una temperatura mínima de $8^{\circ} \mathrm{C}$ y $10^{\circ} \mathrm{C}$ decreciendo en el otoño por cada mes que transcurre, disminuyendo $3^{\circ} \mathrm{C}$ hasta llegar al invierno manteniéndose constante entre $1^{\circ} \mathrm{C}$ y
$2{ }^{\circ} \mathrm{C}$. Se puede observar en el primer bloque 1979-1988 que en el verano la temperatura mínima por mes oscilaba entre $7^{\circ} \mathrm{C}$ y $7.5^{\circ} \mathrm{C}$. Para el segundo bloque 1989-1998 y para el tercer bloque 1999 al 2008 la temperatura mínima promedio oscilaba entre $9^{\circ} \mathrm{C}$ y $10^{\circ} \mathrm{C}$. La variación del incremento con respecto al promedio general en el verano, la temperatura mínima se incrementa $1.2^{\circ} \mathrm{C}$ (Fig. 8). 


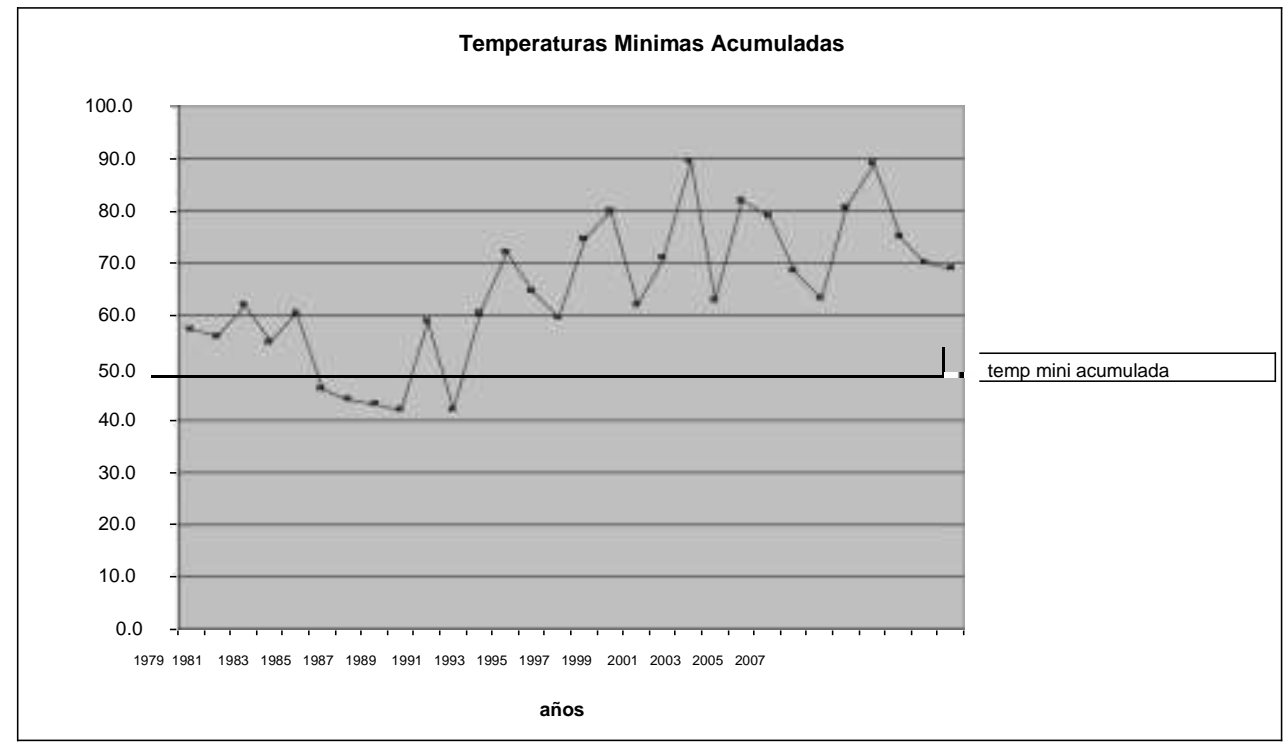

Figura 8. Temperatura mínima acumulada del mismo mes en los años de 1979 - 2009.

El rango de temperatura mínima acumulada está entre $42^{\circ} \mathrm{C}$ y $62^{\circ} \mathrm{C}$ de 1979 a temperatura mínima acumulada fue de $1.2^{\circ} \mathrm{C}$ 1990 y de 1990 al 2003 su incremento de la

Cuadro 1. Precipitación Promedio Mensual por Época del Año.

\begin{tabular}{ccccc}
\hline $\begin{array}{c}\text { Bloque de Años en } \\
\text { Estudio }\end{array}$ & Invierno & Primavera & Verano & Otoño \\
\hline $1979-1988$ & 150 & 250 & 750 & 250 \\
$1989-1998$ & 150 & 250 & 950 & 150 \\
$1999-2008$ & 120 & 280 & 980 & 480 \\
\hline
\end{tabular}


De acuerdo a las precipitaciones promedio mensuales en invierno han disminuido, para la primavera se tiene un incremento de $30 \mathrm{~mm}$, para verano se tiene un incremento de $230 \mathrm{~mm}$ y para otoño se tiene $230 \mathrm{~mm}$.

Cuadro 2. Precipitación Promedio Mensual por Época del Año.

\begin{tabular}{ccccc}
\hline $\begin{array}{c}\text { Bloque de Años en } \\
\text { Estudio }\end{array}$ & Invierno & Primavera & Verano & Otoño \\
\hline $1979-1988$ & 150 & 250 & 750 & 250 \\
$1989-1998$ & 150 & 250 & 950 & 150 \\
$1999-2008$ & 120 & 280 & 980 & 480 \\
\hline
\end{tabular}

\section{CONCLUSIONES}

En los 30 años de estudio de 1979 al 2008 la temperatura promedio máxima oscila entre $24^{\circ} \mathrm{C}$ y $30^{\circ} \mathrm{C}$ donde el periodo de primavera la temperatura oscila entre $\operatorname{los} 28^{\circ} \mathrm{C}$ y $29.6^{\circ} \mathrm{C}$, posteriormente en las estaciones restantes la temperatura varia de $24^{\circ} \mathrm{C}$ a $25^{\circ} \mathrm{C}$. En el primer bloque 1979-1988 la temperatura en el periodo primavera oscila entre $\operatorname{los} 29.6^{\circ} \mathrm{C}$ y $30.3^{\circ} \mathrm{C}$ y el resto del año la temperatura fue de $24^{\circ} \mathrm{C}$ y $25^{\circ} \mathrm{C}$. Es muy semejante a la temperatura promedio de todos los años en estudio. b) De acuerdo al segundo bloque 1989-1998 durante el periodo de primavera la temperatura máxima muestran un decremento de 2 a $4{ }^{\circ} \mathrm{C}$ con respecto al promedio total. Con lo que respecta al tercer bloque 1999-2008 la temperatura máxima empiezan a marcarse a partir del mes de febrero al mes de mayo, donde los meses restantes registran una temperatura máxima promedio entre $25.1^{\circ} \mathrm{C}$ a $25.8^{\circ} \mathrm{C}$. Con respecto al promedio total nos muestra un incremento de $0.5 \mathrm{a} 1^{\circ} \mathrm{C}$. c) De acuerdo a las precipitaciones promedio mensuales que las precipitaciones en invierno han disminuido, para la primavera se tiene un incremento de $30 \mathrm{~mm}$, para verano se tiene un incremento de $230 \mathrm{~mm}$ y para otoño se tiene $230 \mathrm{~mm}$. d) Se debe poner atención cuando la precipitación rebase los $480 \mathrm{~mm}$ en un tiempo de 4 a 6 días las lluvias se han desplazado un mes más y estas terminan en mes de octubre y no en septiembre como en el primer bloque de años.

\section{Revista Científica Biológico Agropecuaria Tuxpan 2 (1)} ISSN: 2007-6940 


\section{LITERATURA CITADA}

Koeppen, W. 1948. Climatología con un estudio de los climas de la tierra. Fondo de cultura económica. Primera edición en español. México-Buenos aires. pp. 199-220.

https://doi.org/10.1086/ahr/82.1.225

Moreno, M. E. E. 1998. Estudio para la integración geográfica del desarrollo agrícola región II Teziutlán. Tesis de licenciatura. Unidad Académica de Ingeniería Agrohidráulica. Benemérita Universidad Autónoma de Puebla. San Juan Acateno. Teziutlán. Puebla. 86 p.

https://doi.org/10.25145/j.pasos.2018.16.052
Romo, G. J. y Arteaga, R. R. 1983. Meteorología Agrícola. Universidad Autónoma Chapingo. Departamento de Irrigación. Chapingo México. pp 94-104. https://doi.org/10.5154/r.inagbi.2009.04.001

Corona, F. J. y Tovar, M, E. 2000. Elementos de Estadística Aplicaciones al método experimental. Editorial trillas México.

\footnotetext{
Copyright (c) 2014 Isaac Villegas Rodiiguez, José Méndez Gómez, Delfino Reyes López, BenjaminBarrios Diaz y César David Torres Femández

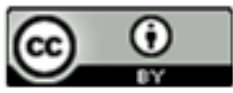

Este texto está proteg ido por una licencia Creative Commora $\underline{4.0}$

Usted es libre para Compartir —copiar y redistribuir el material en cualquier medio of ormato-y Ad aptar el documento —remezclar, transformar y crear a partir del material- para cualquier propósito, inchso para fines comerciales, siempre que cumpla la condición de:

Atribución: Usted debe dar crédito a la obra original de manera adecuada, proporcionar un enlace a la licencia, e indicar si se han realizado cambios. Puede hacerio en cualquier forma razonable, pero no de forma tal que sugiera que tiene el apoyo del licenciante olo recibe por el usoque hace de la obra.

Resumendelicencia - Textocompletodklalicencia
} 